\title{
Endocarditis por Lactococcus garvieae en un paciente sometido a hemodialisis crónica. Primer caso reportado en Chile y revisión de la literatura
}

\author{
Rene Clavero, Juanita Escobar, Sergio Ramos-Avasola, Lorenzo Merello y Francisco Álvarez
}

Lactococcus garvieae endocarditis in a patient undergoing chronic hemodialysis. First case report in Chile and review of the literature

Reports of Lactococcus garvieae infections in humans are scarce, and only one of them in a patient undergoing hemodialysis. We report the first case of Lactococcus garvieae infection in Chile, presenting as an infective endocarditis, ultimately fatal, in a patient with uncomplicated colonic diverticulosis and end stage renal failure undergoing chronic hemodialysis. We review the published cases and discuss the diagnostic and therapeutic challenges associated with this new, increasingly diagnosed pathogen, capable of producing serious infections in susceptible patients.

Key words: Lactococcus garviae infection, infective endocarditis.

Palabras clave: Infección por Lactococcus garvieae, endocarditis infecciosa.

\section{Introducción}

$L$ actococcus garvieae es un patógeno zoonótico emergente, originalmente aislado en mastitis de rumiantes y conocido como responsable de septicemia en peces. Es una cocácea grampositiva, catalasa negativa, anaerobia facultativa que se dispone en pares o cadenas cortas. En base a análisis genéticos fue separada del género Streptococcus el año $1985^{1}$, pero éste aún sigue siendo erróneamente identificado como una variante del género Enterococcus ${ }^{2}$. El género Lactococcus está compuesto por ocho especies, de las cuales Lactococcus lactis y Lactococcus garvieae son los más ampliamente asociados a patologías humanas.

El primer caso reportado por Lactococcus spp. en seres humanos correspondió a una endocarditis infecciosa, publicada el año $1955^{3}$, y desde entonces se han comunicado casos esporádicos. Al parecer este patógeno se comportaría como un agente oportunista de baja virulencia, cuyo principal mecanismo de transmisión estaría asociado al contacto con productos marinos ${ }^{4}$.

Comunicamos el primer caso de infección por Lactococcus garvieae en Chile, que causó una endocarditis en una paciente con falla renal terminal en hemodiálisis crónica y portadora de una diverticulosis de colon.

\section{Caso clínico}

Mujer de 72 años, dueña de casa, con residencia en la ciudad de Viña de Mar, Región de Valparaíso, Chile. Tenía antecedentes de una diabetes mellitus tipo II, hipertensión arterial y una enfermedad renal crónica terminal (ERCT) en hemodiálisis crónica trisemanal hacía cuatro años.

Durante su permanencia en hemodiálisis ambulatoria había presentado problemas con sus accesos vasculares, requiriendo dos fístulas arteriovenosas en ambas extremidades superiores y tres catéteres venosos tunelizados de hemodiálisis. El último en uso, había sido instalado hacía un año y medio, permitiendo mantenerse autovalente durante este tiempo.

Consultó por episodios de calofríos y sensación febril que se presentaban en la primera hora de sesión de hemodiálisis en su centro ambulatorio. Se objetivó una temperatura axilar de $37,8^{\circ} \mathrm{C}$ e hipotensión arterial sintomática de $80 / 50 \mathrm{mmHg}$. No refería baja de peso, sudoración nocturna, fiebre o compromiso del estado general en su domicilio previo a la pesquisa de fiebre durante las sesiones de diálisis. La paciente también negó la ingesta o contacto con productos del mar los días previos a su ingreso al hospital, así como el uso de antiácidos o medicamentos similares.

Se internó en regulares condiciones generales, febril, con temperatura de $38^{\circ} \mathrm{C}$. Al examen cardíaco destacaba
Hospital Dr. Gustavo Fricke, Viña del Mar, Chile (RC, JE). Universidad de Valparaíso, Escuela de Medicina (LM). Universidad Viña del Mar, Escuela de Tecnología Médica, Facultad de Salud (SRA). Universidad Andrés Bello, Escuela de Química y Farmacia, Facultad de Medicina (FA)

Esta revisión no recibió apoyo financiero para su realización. Los autores declaran no tener conflicto de intereses.

Recibido: 11 de octubre de 2016 Aceptado: 16 de mayo de 2017 
un soplo sistólico 2/6 de la punta y al examen pulmonar presentaba crepitaciones en ambas bases pulmonares. El abdomen era normal y no se encontraron fenómenos tromboembólicos en la piel ni en las extremidades. A las ocho horas siguientes al ingreso, se agregó compromiso de conciencia progresivo, con una caída del índice de Glasgow a 10 puntos, sin focalización neurológica. Se sospechó una probable sepsis asociada al catéter de hemodiálisis y dado su agravamiento se trasladó a la Unidad de Cuidados Intensivos (UCI). Los exámenes de laboratorio mostraron una leucocitosis de 20.500 leucocitos $/ \mathrm{mm}^{3}$, con $86 \%$ de neutrófilos, una VHS de $100 \mathrm{mmHg}$ y una anemia normocítica normocrómica con una hemoglobina de 8,5 g/dL. La PCR fue de 39,1 $\mathrm{mg} / \mathrm{dL}$ (valor normal $<1 \mathrm{mg} / \mathrm{dL}$ ). Las pruebas hepáticas fueron normales. El electrocardiograma mostró un ritmo sinusal, con una taquicardia de 110 por min y signos de sobrecarga ventricular izquierda. En la radiografía de tórax tenía signos de congestión pulmonar moderada y

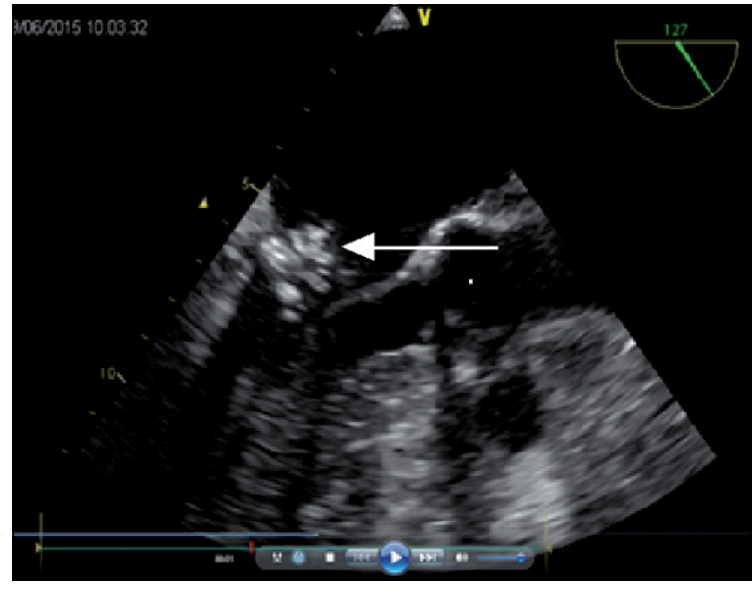

Figura 1. Ecocardiografía 2D que muestra la vegetación móvil situada en el lado arterial de la válvula mitral cerca de la unión mitro-aórtica.

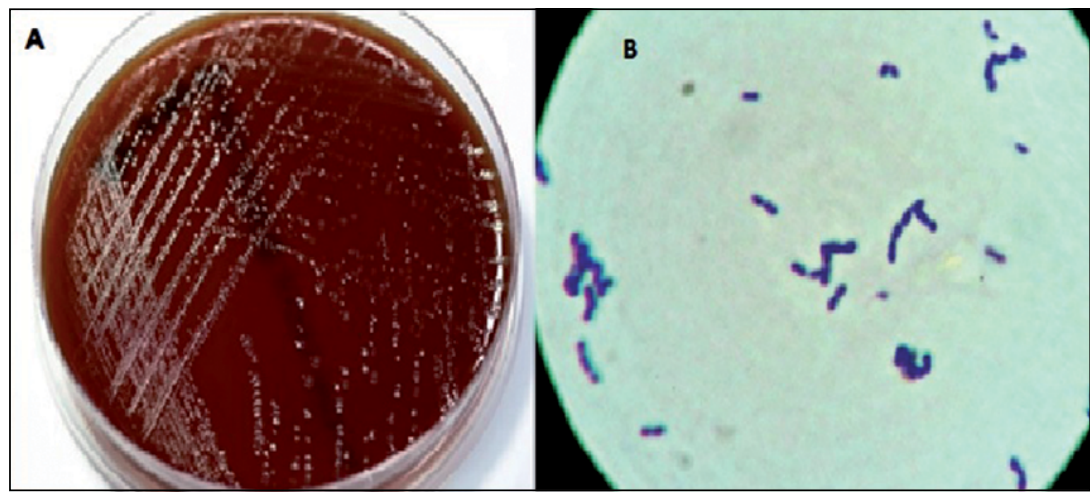

Figura 2. (A) Crecimiento de pequeñas colonias grisáceas de Lactococcus garvieae en una placa de agar sangre después de $24 \mathrm{~h}$ de incubación a $35^{\circ} \mathrm{C}$ en condiciones aeróbicas. (B) Tinción de Gram de Lactococcus garvieae, se aprecian cocáceas grampositivas, dispuestas en cadenas cortas y en pares. crecimiento de las cavidades cardíacas izquierdas. Se tomaron tres hemocultivos periféricos y un hemocultivo a través del catéter tunelizado de hemodiálisis, el cual se retiró y se envió a cultivo con técnica de Maki. Se inició tratamiento antibacteriano empírico con cloxacilina $2 \mathrm{~g}$ iv fraccionado cada $6 \mathrm{~h}$, y amikacina iv 7,5 $\mathrm{mg} / \mathrm{kg}$ post-hemodiálisis. Evolucionó con compromiso multisistémico progresivo con shock séptico y falla respiratoria aguda, requiriendo conexión a ventilación mecánica invasora, uso de aminas vasoactivas (dopamina y noradrenalina) y hemodiálisis convencional diaria. Las TC de cerebro, abdomen y pelvis mostraron cambios degenerativos e involutivos de cerebro y una diverticulosis colónica sin signos de complicación, y sin otros hallazgos significativos.

Al quinto día de hospitalización en UCI se realizó un ecocardiograma 2D transesofágico, donde se constataron vegetaciones de 3 a $4 \mathrm{~mm}$ en ambos velos mitrales asociado a una insuficiencia mitral moderada (Figura 1).

En dos de los hemocultivos periféricos se aislaron cocáceas grampositivas dispuestas en cadenas cortas. En agar soya tripticasa más $5 \%$ sangre de cordero se desarrollaron como colonias grisáceas de tamaño mediano no hemolíticas, semejantes a colonias de Enterococcus (Figura 2). El hemocultivo del catéter de hemodiálisis fue negativo así como el cultivo tipo Maki.

Dado los hallazgos en los cultivos y la presencia de vegetaciones compatibles con una endocarditis infecciosa se ajustó la terapia antibacteriana a vancomicina iv $500 \mathrm{mg}$ asociado a una dosis de carga de gentamicina iv $3 \mathrm{mg} / \mathrm{kg}$, seguido de $1,5 \mathrm{mg} / \mathrm{kg}$ después de cada sesión de hemodiálisis.

Posteriormente, en el laboratorio de microbiología se realizaron pruebas bioquímicas de identificación (hidrólisis de bilis esculina y crecimiento en cloruro de sodio al $6,5 \%$ ) que resultaron positivas; la prueba de catalasa y de movilidad fueron negativas. El sistema automatizado de identificación VITEK-2 Compact (Biomérieux) permitió identificar la cepa como Lactococcus garvieae, con un $98 \%$ de certeza.

La identificación final fue confirmada por el Instituto de Salud Pública de Chile por medio de espectrometría de masas y mediante secuenciación automática de ARN ribosomal 16S.

El estudio de susceptibilidad antimicrobiana por epsilometría con tiras Oxoid, (Hampshire, Inglaterra), entregó una CIM para vancomicina de $2,0 \mu \mathrm{g} / \mathrm{ml}$, cefotaxima de $0,25 \mu \mathrm{g} / \mathrm{ml}$ y para penicilina de $0,5 \mu \mathrm{g} / \mathrm{ml}$. Con el método de difusión Kirby Bauer se midió la sensibilidad a eritromicina, ciprofloxacina, cotrimoxazol y amoxicilina/ácido clavulánico, resultando todos sensibles de acuerdo a los puntos de corte del CLSI-2015. Para clindamicina resultó resistente según los criterios definidos para Streptococcus $\beta$-hemolítico. 
Finalmente, y pesar de las terapias de apoyo implementadas, la evolución posterior fue tórpida falleciendo por una falla orgánica múltiple refractaria, tres semanas después de su ingreso al centro asistencial.

\section{Discusión}

Lactococcus garvieae se describe como un patógeno oportunista y emergente; sin embargo, es frecuentemente confundido con Enterococcus spp., por lo que más que un microorganismo emergente quizás lo más adecuado sea hablar de un patógeno erróneamente identificado.

Al realizar una búsqueda bibliográfica actualizada es posible encontrar 31 casos de infecciones donde Lactococcus spp. fue identificado como el agente causal (Tabla 1). De éstos $42 \%$ provienen de Europa, 35\% de Asia y 23\% de América. Según el tipo de infección, hubo 20 endocarditis infecciosas, (11 de válvulas mitrales nativas ${ }^{4-12}$, tres de prótesis de válvula mitral ${ }^{7,13,14}$, tres válvulas aórticas nativas $^{2,12,15}$, dos prótesis de válvula aórtica ${ }^{9,16}$ y una válvula tricúspidea ${ }^{17}$ ), dos casos de bacteriemia ${ }^{4,18}$, dos casos de septicemia ${ }^{4,19}$ y dos casos de peritonitis ${ }^{4,20}$. Otros fueron casos aislados de absceso hepático ${ }^{21}$, osteomielitis ${ }^{22}$, colecistitis aguda ${ }^{23}$, espondilitis ${ }^{24}$, infección de prótesis de cadera $^{25}$ y un hematoma subdural ${ }^{9}$. La mayoría de los casos se encuentran entre los 40 y 85 años de edad con un solo caso pediátrico, de un paciente de 10 años.

Con respecto al género, el número de casos fue similar entre hombres y mujeres (12 y 16 casos, respectivamente). En la mayoría de los pacientes se describe algún antecedente médico asociado, como implante de marcapasos y prótesis valvulares, cirugía coronaria, periodontitis, linfoma, terapia de antiácidos o anticoagulantes y patología digestiva como estenosis esofágica, úlcera gástrica, perforación duodenal, diverticulosis, pólipos y cirugía de cáncer colorrectal.

Según Chan ${ }^{24}$ y cols., el tracto digestivo presentaría un disturbio fisiológico asociado a cirugías previas, patologías activas, o el uso de terapia con antiácidos o inmunosupresores, lo que condicionaría a que el microorganismo pudiera migrar a otras regiones, donde se produciría la infección final. En los casos revisados, la infección más frecuente fue la endocarditis infecciosa (20/31, 64\%).

El mecanismo más probable de infección es el contacto con productos del mar, ya sea por alguna actividad relacionada con dichos productos (chef, vendedor de pescados, trabajadores de granjas de cultivos de peces, etc.) o por la ingesta directa de productos del mar principalmente crudos $^{4}$. En esta revisión en 11 de los 31 pacientes había antecedente de una actividad relacionada con productos del mar o su ingesta, en 12 pacientes no se consignó este dato y en los restantes ocho casos se reportó otro factor de riesgo. Así parece relevante obtener la información sobre contacto con productos del mar por actividad laboral o por consumo, al momento de plantearse su diagnóstico.

Siendo en general una bacteria con poca virulencia, puede producir infecciones graves en pacientes con alguna condición predisponente, como la presencia de marcapasos, valvulopatías, prótesis valvulares, etc. Así, en $80 \%$ de los casos revisados se consigna alguna condición predisponente.

En la infección por L. garvieae se ha descrito una mortalidad variable. Chan ${ }^{24}$ y cols., realizaron una revisión de nueve casos publicados encontrando tres fallecidos, es decir, 33\% de mortalidad. Otra revisión ${ }^{8}$ de 21 casos, de los cuales se tuvo la información precisa del desenlace en 18 casos, tres fallecieron, es decir, $16 \%$ de mortalidad. A la fecha, sumando nuestro caso a lo publicado en la literatura médica, se puede describir un $21 \%$ de mortalidad.

La patogenicidad e infectividad de L. garvieae no están del todo claras. Se sabe que no forma parte de la microbiota del sistema digestivo y que el consumo de leche, peces o alimentos manufacturados contaminados podrían ser una fuente de infección. Por otra parte la acidez gástrica, enzimas pancreáticas, secreciones biliares e intestinales, movimientos peristálticos y la integridad de las células epiteliales son factores protectores de la infección. La existencia de algún trastorno gastrointestinal como úlceras, pólipos, o divertículos, podría actuar como factor predisponente de la traslocación bacteriana.

En este caso no se pudo documentar la ingesta reciente de productos del mar (pescados o mariscos) o antiácidos, además es muy improbable que la diálisis por sí misma haya mediado o facilitado la infección puesto que no hubo signos de infección de los accesos y el cultivo de catéter resultó negativo. En uno de los exámenes imagenológicos se documentó la existencia de una diverticulosis colónica, que se ha asociado en otros casos. No obstante lo anterior, con los datos disponibles no se puede confirmar esta hipótesis.

Del punto de vista microbiológico, la identificación de L. garvieae presenta dos desafíos: primero es muy común que este patógeno sea erróneamente identificado como Enterococcus spp. ya que ambos tienen similares características morfológicas ${ }^{26,27}$. Además son catalasa negativa, PYR positivo, crecen en suero fisiológico al $6,5 \%$, y pueden hidrolizar la esculina. La principal diferencia entre ambos es que Lactococcus es sorbitol negativo y Enterococcus es positivo ${ }^{10}$. Actualmente uno de los métodos de identificación más rápido y confiable para la identificación de L. garvieae es a través de espectrometría de masas, asistida por láser (MALDI-TOF MS). Estos resultados posteriormente deberían ser confirmados molecularmente mediante la secuenciación del gen $\mathrm{ARNr} 16 \mathrm{~s}^{15}$.

El segundo desafío es la diferenciación entre las especies garvieae y lactis. Para ello es útil la prueba de susceptibilidad a clindamicina, en que $L$. garvieae es resistente y L. lactis es sensible. 


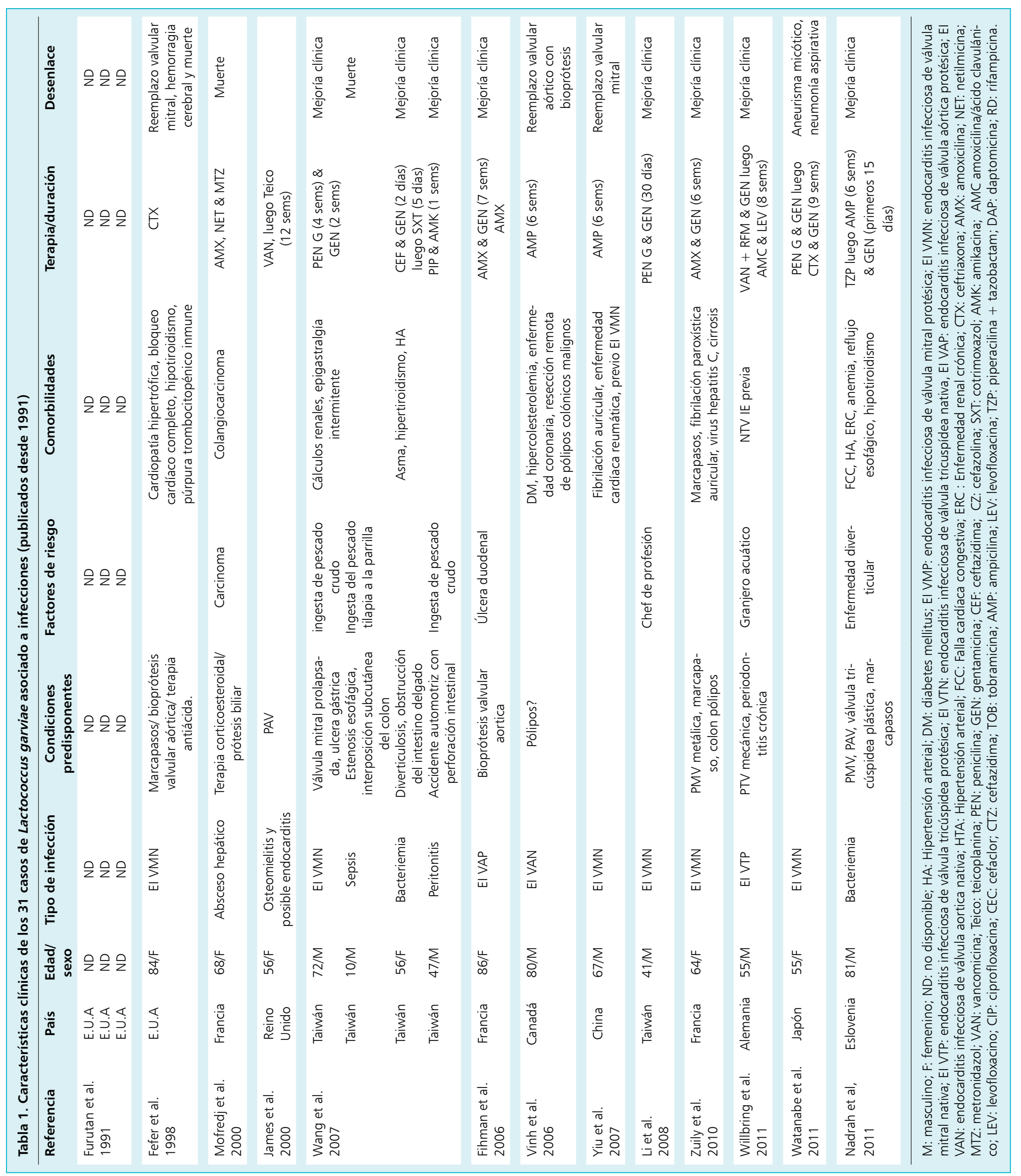




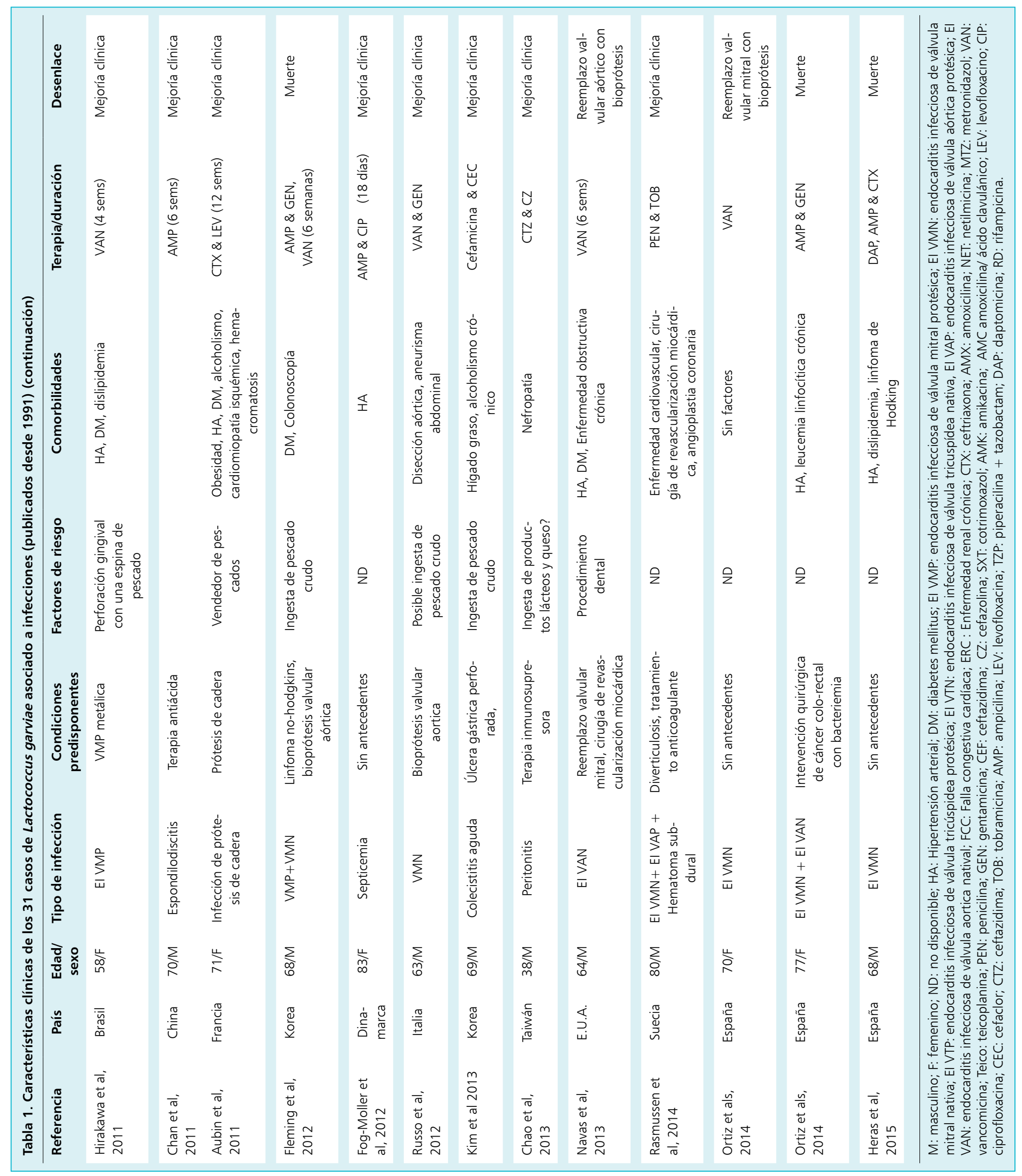


Tabla 2. Esquemas antimicrobianos de los 31 casos de Lactococcus garviae asociado a infecciones (publicados desde 1991)

\begin{tabular}{|c|c|c|c|c|c|}
\hline Referencias & n de casos & Tipo de infección & Terapia antimicrobiana 1 & Terapia antimicrobiana 2 & Fallecidos \\
\hline Fefer et al. & 1 & Endocarditis infecciosa & $\mathrm{CZ}+\mathrm{VAN}$ & CTX & 1 \\
\hline Wang et al.; Li et al. & 2 & Endocarditis infecciosa & PEN & GEN & \\
\hline Fihman et al.; Zuily et al. & 2 & Endocarditis infecciosa & $A M X+G E N$ & & \\
\hline Vinh et al.; Yiu et al & 2 & Endocarditis infecciosa & AMP & & \\
\hline Wilbrig et al. & 1 & Endocarditis infecciosa & VAN + GEN & $A M C+L E V$ & \\
\hline Watanabe et al. & 1 & Endocarditis infecciosa & PEN + GEN & $\mathrm{CTX}+\mathrm{GEN}$ & \\
\hline Hirakawa et al.; Navas et al.; Ortiz et al. & 3 & Endocarditis infecciosa & VAN & & \\
\hline Ortiz et al. & 1 & Endocarditis infecciosa & $\mathrm{AMP}+\mathrm{GEN}$ & & 1 \\
\hline Fleming et al. & 1 & Endocarditis infecciosa & $\mathrm{AMP}+\mathrm{GEN}$ & VAN & 1 \\
\hline Russo et al. & 1 & Endocarditis infecciosa & VAN + GEN & & \\
\hline Rassmussen et al. & 1 & Endocarditis infecciosa & $\mathrm{PEN}+\mathrm{TOB}$ & & \\
\hline Heras et al. & 1 & Endocarditis infecciosa & $\mathrm{DAP}+\mathrm{AMP}$ & CTX & 1 \\
\hline Mofredj et al. & 1 & Absceso hepático & $\mathrm{AMX}+\mathrm{NET}+\mathrm{MTZ}$ & & 1 \\
\hline Wang et al.; Chao et al. & 2 & Peritonitis & $\mathrm{PIP}+\mathrm{AMK}$ & & \\
\hline Wang et al. & 1 & Bacteriemia & $\mathrm{CEF}+\mathrm{GEN}$ & STX & \\
\hline Nadrah et al. & 1 & Bacteriemia & TZP & $\mathrm{AMP}+\mathrm{GEN}$ & \\
\hline Fog-Moller et al. & 1 & Septicemia & $\mathrm{AMP}+\mathrm{CIP}$ & & \\
\hline James et al. & 1 & Osteomielitis & VAN & Teico & \\
\hline Chan et al. & 1 & Espondilodiscitis & AMP & & \\
\hline Aubin et al & 1 & Infección prótesis cadera & $C T X+L E V$ & & \\
\hline Kim et al. & 1 & Colecistitis aguda & CEC + cefamicina & & \\
\hline Rassmussen et al. & 1 & Hematoma subdural & $\mathrm{PEN}+\mathrm{TOB}$ & & \\
\hline Furutan et al. & 3 & ND & ND & ND & \\
\hline
\end{tabular}

El tratamiento antimicrobiano recomendado es variable. Es así como en los casos reportados de endocarditis infecciosa se utilizaron hasta 10 esquemas antimicrobianos distintos (Tabla 2), con cambio del esquema terapéutico inicial en $50 \%$ de ellos. Una probable causa de esta variabilidad puede ser la falta de criterios formales para evaluar la susceptibilidad antimicrobiana de este microorganismo, lo que induce a iniciar el tratamiento en forma empírica.

\section{Conclusión}

Esta comunicación alerta sobre la presencia de un nuevo patógeno, cada vez más diagnosticado, capaz de producir infecciones graves y potencialmente letales en pacientes susceptibles. Además pone en evidencia la ne- cesidad de definir tratamientos antibacterianos específicos para infecciones por L. garviae.

\section{Resumen}

Los casos reportados de infección por Lactococcus garvieae son escasos y sólo uno asociado a hemodiálisis. Comunicamos el caso de endocarditis infecciosa de curso fatal por L. garvieae en un paciente con una enfermedad renal crónica sometido a hemodiálisis y portador de diverticulosis colónica no complicada. Se realiza una revisión de los casos publicados y se discuten los actuales desafíos diagnósticos y terapéuticos de este patógeno, capaz de producir infecciones graves y potencialmente fatales en pacientes susceptibles. Este sería el segundo caso de infección asociada a hemodiálisis y el primero reportado en Chile. 


\section{Referencias bibliográficas}

1.- Schleifer K, Kraus J, Dvorak C, KilpperBälz R, Collins M, Fischer W. Transfer of Streptococcus lactis and related Streptococci to the genus Lactococcus gen. nov. Syst Appl Microbiol 1985; 6: 183-95.

2.- Vinh D C, Nichol K A, Rand F, Embil J M. Native-valve bacterial endocarditis caused by Lactococcus garvieae. Diagn Microbiol Infect Dis 2006; 56: 91-4.

3.- Wood H F, Jacobs K, McCarty M. Streptococcus lactis isolated from a patient with subacute. Am J Med 1955; 18: 345-7.

4.- Wang C Y, Shie H S, Chen S C, Huang P, Hsieh I C, Wen M S, et al. Lactococcus garvieae infections in humans: possible association with aquaculture outbreaks. Int $\mathrm{J}$ Clin Pract 2007; 61: 68-73.

5.- Fefer J J, Ratzan K R, Sharp S E, Saiz E. Lactococcus garvieae endocarditis: report of a case and review of the literature. Diagn Microbiol Infect Dis 1998; 32: 127-30.

6.- Watanabe Y, Naito T, Kikuchi K, Amari Y, Uehara $\mathrm{Y}$, Isonuma $\mathrm{H}$, et al. Infective endocarditis with Lactococcus garvieae in Japan: a case report. J Med Case Rep 2011; 5: 356.

7.- Fleming H, Fowler S V, Nguyen L, Hofinger D M . Lactococcus garvieae multivalve infective endocarditis in a traveler returning from South Korea. Travel Med Infect Dis 2012; 10: 101-4.

8.- Russo G, Iannetta M, D’Abramo A, Mascellino M, Pantosti A, Erario L, et al. Lactococcus garvieae endocarditis in a patient with colonic diverticulosis: first case report in Italy and review of the literature. New Microbiol 2012; 35: 495-501.

9.- Rasmussen M, Björk Werner J, Dolk M, Christensson B. Lactococcus garvieae endocarditis presenting with subdural haematoma. BMC Cardiovasc Disord 2014; 14: 13.

10.- Heras Cañas V, Pérez Ramírez M D, Bermudez Jiménez F, Rojo Martin M D, Miranda Casas C, Marin Arriaza M, et al. Lactococcus garvieae endocarditis in a native valve identified by MALDI-TOF MS and PCR-based 16s rRNA in Spain: a case report. New Microbes New Infect 2015; 5: 13-5.

11.- Yiu K H, Siu C W, To K K, Jim M H, Lee $\mathrm{K} \mathrm{L}$, Lau C P, et al. A rare cause of infective endocarditis; Lactococcus garvieae. Int J Cardiol 2007; 114: 286-7.

12.- Ortiz C, López J, del Amo E, Sevilla T, García P, San Román J. Endocarditis infecciosa por Lactococcus garvieae: presentación de 2 casos y revisión de la literatura. Rev Española Cardiol 2014; 67: 776-8

13.- Zuily S, Mami Z, Meune C. Lactococcus garvieae endocarditis. Arch Cardiovasc Dis. 2011; 104: 138-9.

14.- Hirakawa T, Alves da Costa F, Cairo Vilela M, Rigon M, Abensur H, Elmor de Araújo M R. Endocarditis por Lactococcus garvieae: primer relato de caso de América Latina. Arq Bras Cardiol 2011; 97: 108-10.

15.- Navas M E, Hall G, EI Bejjani D. A case of endocarditis caused by Lactococcus garvieae and suggested methods for identification. J Clin Microbiol 2013; 51: 1990-2.

16.- Fihman V, Raskine L, Barrou Z, Kiffel C, Riahi J, Berçot B, et al. Lactococcus garvieae endocarditis: identification by $16 \mathrm{~S}$ rRNA and sodA sequence analysis. J Infect 2006; 52 : e3-6.

17.- Wilbring M, Alexiou K, Reichenspurner $\mathrm{H}$, Matschke K, Tugtekin S M. Lactococcus garvieae causing zoonotic prosthetic valve endocarditis. Clin Res Cardiol 2011; 100: 5456.

18.- Nadrah K, Cerar T, Papst L, Volkar-Meglič J, Matičič M, Karner P, et al. Lactococcus garvieae septicaemia in a patient with artificial heart valves. Wien Klin Wochenschr 2011; 123 (21-22): 677-9.

19.- Fog-Møller T, Andersen J. Serious infection with Lactococcus garvieae. Ugeskr Laeger 2012; 174: 1096-7.

20.- Chao C T, Lai C F, Huang J W. Lactococcus garvieae peritoneal dialysis peritonitis. Perit Dial Int2 013; 33: 100-1.

21.- Mofredj A, Baraka D, Kloeti G, Dumont J L. Lactococcus garviae septicemia with liver abscess in an immunosuppressed patient. Am J Med 2000; 109: 513-4.

22.- James P R, Hardman S M, Patterson D L. Osteomyelitis and possible endocarditis secondary to Lactococcus garvieae: a first case report. Postgrad Med J 2000; 76: 301-3.

23.- Kim J H, Go J, Cho C R, Kim J I, Lee M S, Park S C. First report of human acute acalculous cholecystitis caused by the fish pathogen Lactococcus garvieae. J Clin Microbiol 2013; 51: 712-4.

24.- Chan J, Woo P, Teng J, Lau S, Leung S, Tam F, et al. Primary infective spondylodiscitis caused by Lactococcus garvieae and a review of human L. garvieae infections. Infection 2011; 39: 259-64.

25.- Aubin G G, Bémer P, Guillouzouic A, Crémet L, Touchais S, Fraquet N, et al. First report of a hip prosthetic and joint infection caused by Lactococcus garvieae in a woman fishmonger. J Clin Microbiol 2011; 49: 2074-6.

26.- Facklam R, Elliott J A. Identification, classification, and clinical relevance of catalasenegative, gram-positive cocci, excluding the streptococci and enterococci. Clin Microbiol Rev 1995; 8: 479-95.

27.- Elliott J A, Collins M D, Pigott N E, Facklam R R. Differentiation of Lactococcus lactis and Lactococcus garvieae from humans by comparison of whole-cell protein patterns. J Clin Microbiol 1991; 29: 2731-4. 